\title{
Aplicación del método Scoring para la clasificación integral de clientes
}

\author{
Application of the Scoring method for integral customer classification \\ Rosario C. Garza Ríos ${ }^{1} \quad$ Caridad N. González Sánchez $\quad$ Ernesto L. Rodríguez González $3 *$ \\ Recibido 28 de septiembre de 2017, aceptado 18 de julio de 2018. \\ Received; September 28, 2017 Accepted: July 18, 2018
}

\begin{abstract}
RESUMEN
Existen entidades que brindan servicios in situ, donde el intercambio de valor ocurre en las instalaciones del cliente, involucrando para ello un número mayor de recursos.

En el trabajo se presentan dos entidades, una oferta sistemas de seguridad "llave en mano", ejecutando los trabajos de proyección, suministros, instalación y puesta en marcha, adiestramiento al cliente y el posterior servicio de mantenimiento de los sistemas, la otra distribuye helados a diferentes puntos de venta. En ambas entidades los clientes son clasificados considerando solamente un criterio (tecnología instalada y distancia).

Ambas entidades han presentado problemas con los indicadores de desempeño, siendo necesario el rediseño de sus sistemas de planificación. El primer paso que se propone para la conformación del plan es realizar la clasificación integral de los clientes teniendo en cuenta un conjunto de criterios.

Se muestra cómo obtener dicha clasificación utilizando un enfoque multicriterial específicamente el método Scoring, el cual permitió de forma rápida y eficiente determinar la clasificación integral de los clientes en las entidades objeto de estudio.

Entre los resultados económicos se pueden mencionar: un ahorro en 11760 litros de petróleo anuales, lo que redunda en un ahorro de \$ 5 576, 64 USD para una entidad de servicio llave en mano, mientras que para la entidad distribuidora de helado aumenta el ingreso en $\$ 35347$, la utilidad en $\$ 40588,45$ y el costo disminuye en $\$ 5470$ para un mes de aplicación del procedimiento.
\end{abstract}

Palabras clave: Clasificación, multicriterio, suma ponderada, sistemas de seguridad, clúster.

\begin{abstract}
There are entities that provide on-site services, where the exchange of value occurs in the client's facilities, involving a greater number of resources.

In the work, two entities are presented, an offer "turnkey" security system, executing projection, supplies, installation and commissioning, training the client and the subsequent maintenance service of the systems, the other distributes ice cream to different points of sale. In both entities the clients are classified considering only one criterion (installed technology and distance).

Both entities have presented problems with the performance indicators, being necessary the redesign of their planning systems. The first step that is proposed for the conformation of the plan is to carry out the integral classification of the clients taking into account a set of criteria.
\end{abstract}

1 Facultad de Ingeniería Industrial. Universidad Tecnológica de la Habana José Antonio Echeverría (CUJAE). Dirección postal: Calle 114 N $^{\circ} 11901$ entre Ciclovía y Rotonda. Marianao. La Habana. Cuba. E-mail: rosariog@ind.cujae.edu.cu

2 Centro de Estudios de Matemática para las Ciencias Técnicas (CEMAT). Universidad Tecnológica de la Habana José Antonio Echeverría (CUJAE). Calle 114 Nº 11901 entre Ciclovía y Rotonda. Marianao. La Habana, Cuba. E-mail: caryg @ cemat.cujae.edu.cu

3 Facultad de Ingeniería. Universidad Panamericana. Campos México. C Augusto Rodin 498, Colonia Insurgentes Mixcoac, Benito Juárez. Ciudad de México, México. E- mail: erodrigo@up.edu.mx

* Autor de correspondencia: erodrigo@up.edu.mx 
It is showned how to obtain this classification using a multicriterial approach, specifically the Scoring method, which allowed to quickly and efficiently determine the integral classification of clients in the entities under study.

Among the economic results can be mentioned: a saving in 11760 liters of oil per year, which results in a savings of \$ 5 576, 64 USD for a turnkey service entity, while for the ice cream distribution entity increases the income at $\$ 35,347$, the utility at $\$ 40,588.45$ and the cost decreases at $\$ 5,470$ for one month of application of the procedure.

Keywords: Classification, multicriteria, weighted sum, security system, cluster.

\section{INTRODUCCIÓN}

Uno de los tres sectores en que se clasifican las actividades económicas son los servicios los cuales cada día alcanzan mayor relevancia. En Cuba, más del 80\% del PIB lo aportan las entidades de servicio, y el desarrollo vertiginoso en esta esfera ha provocado que los empresarios dedicados a esta actividad no solo se ocupen de satisfacer a los clientes que ya poseen, sino que también traten de captar otros.

Un servicio constituye el uso y aplicación de recursos en beneficio de otros o de la propia entidad [2, 9-10, 14]. Existen empresas donde el cliente debe acudir a las instalaciones para recibir el servicio (restaurantes, hospitales, bancos, oficinas de correos, entre otras), y servicios con base in situ, donde el prestador del servicio es el que se mueve para brindar el mismo, por ejemplo, la reparación de efectos electrodomésticos.

Para brindar servicios de calidad se utilizan diferentes técnicas y procedimientos en correspondencia con las características del mismo, fundamentalmente de ayuda a la toma de decisiones entre las que se destacan: simulación, programación lineal, análisis clúster, técnicas multicriteriales entre otras $[2,8,12]$.

En el presente trabajo se exponen dos entidades que ofertan servicio in situ, una oferta servicios de llave en mano realizando los trabajos de instalación puesta en marcha, mantenimiento preventivo y correctivo de equipos de seguridad y protección. La otra entidad oferta servicio de distribución de helados.

Ambas entidades han presentado problemas en la utilización de sus recursos con un incremento de los gastos y un servicio ineficiente a los clientes.

Una de las acciones a realizar para mejorar el servicio ofertado en ambas entidades e incrementar la utilización de los recursos, es la clasificación de los clientes lo cual contribuirá a la disminución de los gastos de transportación.

La clasificación integral de los clientes en opinión de los autores es un problema multicriterio en espacios discretos $[11,13,17]$, dado que se quiere clasificar al mismo considerando un conjunto de criterios que están presentes en cada tecnología, definiendo la importancia de cada uno dentro de la misma y la incidencia de cada tecnología en el tiempo de servicio de mantenimiento, lo cual será utilizado para la confección del plan. En este trabajo se selecciona el método Scoring para resolver este problema por su manera rápida y sencilla de obtener la solución.

\section{DESCRIPCIÓN DE LAS ENTIDADES OBJETO DE ESTUDIO}

\section{Entidad llave en Mano}

Esta entidad planifica el mantenimiento preventivo a sus clientes independientemente de las tecnologías instaladas (protecciones eléctricas (protección contra descargas atmosféricas, tierra física, supresores de transientes y cableado estructurado), seguridad electrónica (sistemas de alarmas contra intrusos, sistemas de control de acceso y circulación y, sistemas de circuito cerrado de televisión), extinción fija (extinción por agua, gases o cualquier otro agente extintor) y extinción portátil (extintores y carretillas)). Para ello, solo considera la fecha del último mantenimiento y la frecuencia requerida para el mantenimiento, teniendo en cuenta la tecnología instalada. Se han presentado dificultades en el cumplimiento del plan de mantenimiento lo que ha motivado un alto consumo de combustible fósil.

El mantenimiento se realiza por brigadas especializadas las cuales atienden siempre a los mismos clientes independientemente de la tecnología 
instalada. Un estudio anterior permitió detectar que la distribución territorial de los clientes y su asignación a las brigadas de mantenimiento no cumplen con los objetivos de la empresa, debido al alto consumo de combustible y es por eso que se hace necesaria la búsqueda de alternativas que permitan realizar una nueva distribución con el objetivo de obtener un mayor ahorro e incrementar las ganancias. En la Figura 1 se muestra la distribución de los clientes obtenidas en el estudio anterior donde se observa una inadecuada distribución de los mismos.

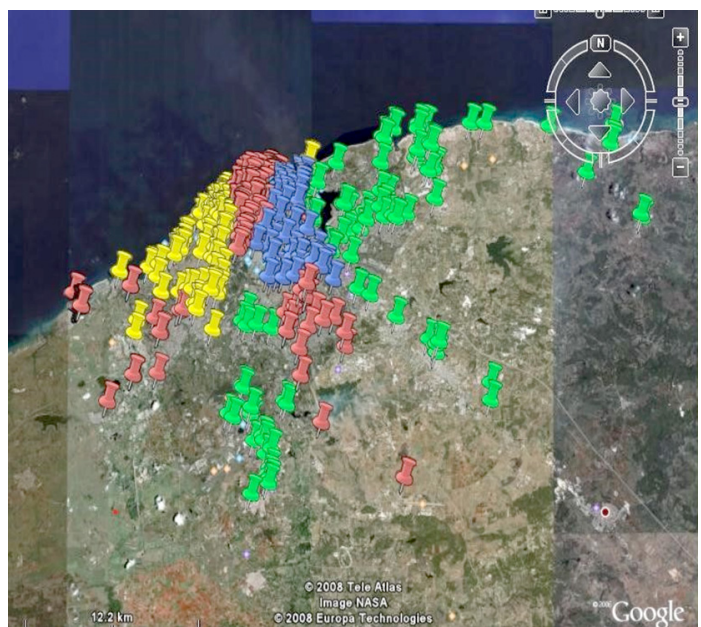

Figura 1. Distribución de los clientes.

La aplicación del análisis clúster permitió obtener una mejor distribución de los clientes entre las brigadas, lo que garantizó obtener los resultados que se muestran en la Figura 2.

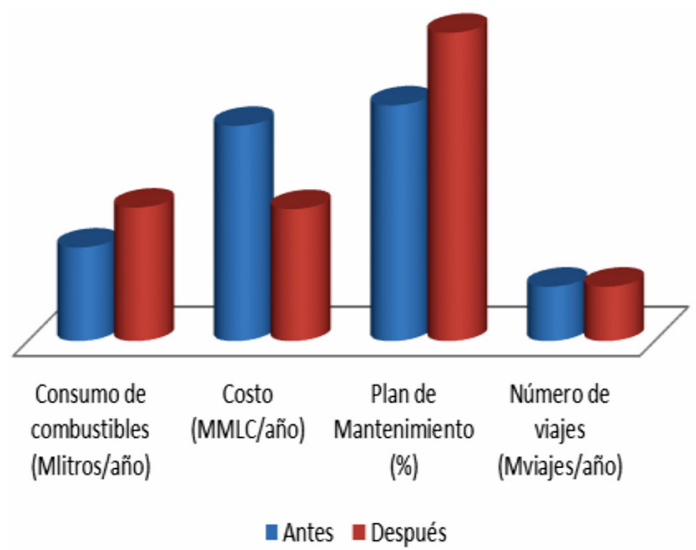

Figura 2. Comparación de indicadores de desempeño.
La nueva distribución obtenida mejora los indicadores de desempeño analizados, sin embargo aún existen incumplimientos en el plan de mantenimiento y se mantiene el número de viajes a realizar. Esto se debe a que la entidad cuenta con un total de 2 132 clientes. De ellos el 47\% (1:310 clientes) tiene instaladas al menos 2 de las tecnologías que brinda, lo que provoca que un cliente deba ser visitado más de una vez en el mes.

La falta de integración de tecnologías en el servicio de mantenimiento correctivo, ha provocado incumplimiento de los planes en los meses planificados, para un cumplimiento promedio de un $38,4 \%, 59,3 \%$ y $73,4 \%$ en los últimos años. Así mismo, se incurre en gastos por concepto de consumo de combustible de \$3:600 anuales, al tener que visitar a un cliente al menos tres veces en un mes [1].

La planificación actual de los mantenimientos además de ocasionar pérdidas en la entidad, conlleva a una subutilización de los recursos humanos y materiales, detectándose un desaprovechamiento de la jornada laboral del $30 \%$, provocando además la insatisfacción por parte del $68 \%$ de los clientes, que considera que el servicio ofertado es Regular.

La entidad posee una clasificación de sus clientes confeccionada de forma empírica, la cual no se sustenta en un basamento científico.

En ningún criterio de clasificación se mencionan elementos que caractericen a los clientes, lo que lleva a pensar que estos no influyen en la complejidad del mismo.

\section{Entidad de distribución de helados}

La fábrica de helados objeto de estudio se encarga de producir y distribuir helados de alta calidad. En estos momentos la fábrica brinda alrededor de 30 sabores y 7 formas de presentación.

La empresa tiene como compromiso satisfacer las necesidades de los clientes con la máxima calidad garantizando que sus productos mantengan la marca estatal de calidad y lograr que los nuevos sabores que se produzcan también alcancen dicha categoría.

Los resultados de esta estrategia han sido efectivos en cuanto a la calidad del helado. Este aspecto ha 
logrado diferenciar este producto de los competidores y lo ha colocado como líder del mercado nacional; sin embargo, presenta grandes dificultades en el servicio de distribución, lo que está afectando su imagen en el mercado del helado, por no contar con una herramienta que le permita realizar un buen enrutamiento, con lo que se lograría minimizar el tiempo, el gasto de combustible y por ende, el gasto en moneda libremente convertible y aumentar el nivel de servicio y la utilidad de la fábrica.

En un estudio anterior se determinó el nivel de servicio ofertado por la fábrica de helados, los resultados obtenidos fueron poco alentadores, siendo el nivel de servicio total de $17.08 \%$, estando afectado éste no solamente por el plazo de entrega sino también por la satisfacción de los pedidos.

Complementando este análisis se efectuó una encuesta a 68 clientes (puntos de venta), lo que representó el 34\% del total; entre los resultados obtenidos se encuentra la respuesta referida a la pregunta: valoración del servicio de distribución, el procesamiento de esta pregunta arrojó que el servicio que oferta la fábrica a sus clientes se puede calificar de bajo, dado porque la mayoría, el 85,21\%, valora este entre Regular y Mal, enfatizando el problema en los plazos de entrega, donde el $66 \%$ de los clientes plantea que generalmente reciben el pedido fuera del horario acordado e incluso hay clientes que no lo reciben.

Estos resultados dan una medida de los atrasos, motivados por un conjunto de problemas que hace que no todos los clientes que necesiten de este servicio puedan ser satisfechos en el momento oportuno. Entre estos problemas están: no existe la cantidad de productos suficiente para satisfacer todos los pedidos, o no hay la capacidad de transporte necesaria para realizar la distribución. Los clientes se encuentran agrupados en 19 rutas establecidas las cuales se realizaron de forma empírica, manteniéndolas sin variación a pesar de que han existido cambios en las condiciones. Se hace necesario una nueva clasificación de los clientes para determinar nuevas rutas de distribución que incrementen la eficiencia de este proceso.

Para mejorar el plan de mantenimiento preventivo y la distribución de helados es necesario en primer lugar, determinar la clasificación de los clientes siendo este el problema que se pretende resolver en el presente trabajo.

Para dar solución al problema planteado se traza como hipótesis: El uso de técnicas multicriteriales específicamente el método Scoring permite realizar de forma rápida y eficiente la clasificación de los clientes disminuyendo los costos y permitiendo incrementar la cantidad de clientes visitados.

\section{CLASIFICACIÓN DE CLIENTES CON ENFOQUE MULTICRITERIAL}

Los autores proponen para la clasificación de los clientes con enfoque multicriterio, utilizar el procedimiento descrito por Aragonés [11] con algunas modificaciones para adaptarlo a las características del problema. Los pasos se muestran a continuación:

1. Seleccionar el grupo de experto que participará en el proceso de clasificación.

2. Seleccionar los criterios (representar el árbol jerárquico de los objetivos).

3. Ponderación de los criterios (asignación de pesos).

4. Determinación de la escala a utilizar para la clasificación de los clientes.

5. Valoración de las alternativas: en este caso las alternativas serán los clientes a clasificar y lo que se realizará es la determinación para cada cliente del valor que recibirá en cada criterio.

6. Aplicación de una técnica para el ordenamiento o selección, en este caso lo que se realizará es el cálculo de la evaluación final.

7. Determinación de la escala y la clasificación de los clientes.

A continuación, se desarrollarán los pasos del procedimiento que en opinión de los autores son los más representativos para ambas entidades:

\section{Llave en mano}

\section{Paso 2: Selección de los criterios.}

Como el objetivo de este trabajo es diseñar un sistema para la clasificación integral de los clientes, la selección de los criterios depende de la tecnología que estos tienen instaladas, se consideró como criterios del primer nivel de jerarquía las cuatro tecnologías a las que la entidad les da mantenimiento. En la Figura 3 se muestra el árbol jerárquico obtenido después de la realización del trabajo grupal para su determinación. 


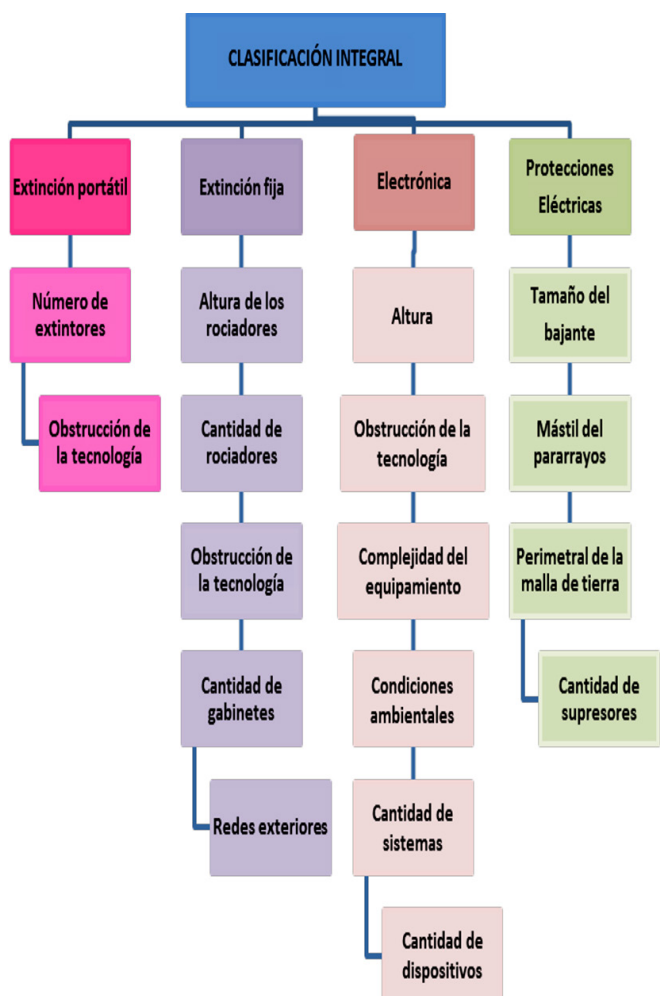

Figura 3. Árbol jerárquico de los criterios.

Obtenidos los criterios a valorar para la clasificación integral de los clientes, se realiza un trabajo en grupo con el objetivo de determinar la ponderación de cada uno de estos criterios así como la determinación de la escala con que se trabajará para evaluar a cada cliente, lo que corresponde a los pasos 3 y 4 del procedimiento utilizado.

Pasos 3 y 4: Ponderación de los criterios (asignación de pesos) y determinación de la escala a utilizar para la clasificación de los clientes.

Para la obtención de los pesos se utilizó el método Delphi en este caso modificado, pues no se realizan las rondas 1 y 2 , debido a que los criterios ya habían sido definidos anteriormente por los técnicos y especialistas. Para procesar la votación de los expertos y llegar a conformar los pesos de los mismos, se decide aplicar el método Rating [7], el cual integra los intereses de cada miembro del grupo en un modelo más efectivo. Otros métodos se pueden encontrar en [4-6].

La escala utilizada para la realización de la votación por parte de los miembros del grupo fue entre $1 \mathrm{y} 4$, donde 4 representa el criterio con mayor importancia, y 1, el criterio menos importante [11]. Los pesos obtenidos una vez realizada la votación se muestran en las Tablas de la 1 a la 3.

Tabla 1. Pesos para la tecnología electrónica.

\begin{tabular}{|c|c|c|c|c|c|c|}
\cline { 2 - 7 } \multicolumn{1}{c|}{} & \multicolumn{6}{c|}{ Electrónica } \\
\hline \multirow{2}{*}{ Peso } & \multicolumn{6}{c|}{0,40} \\
\cline { 2 - 7 } & A & OT & CE & CA & CS & CD \\
\hline Peso & 0,12 & 0,15 & 0,25 & 0,14 & 0,16 & 0,18 \\
\hline
\end{tabular}

Tabla 2. Pesos para protecciones eléctricas.

\begin{tabular}{|c|c|c|c|c|}
\cline { 2 - 5 } \multicolumn{1}{c|}{} & \multicolumn{4}{c|}{ Protecciones eléctricas } \\
\hline \multirow{2}{*}{ Peso } & \multicolumn{3}{|c|}{0,20} & \\
\cline { 2 - 5 } & TB & MP & PMT & CS \\
\hline Peso & 0,17 & 0,22 & 0,33 & 0,28 \\
\hline
\end{tabular}

Tabla 3. Pesos para extinción portátil y fija.

\begin{tabular}{|c|c|c|c|c|c|c|c|}
\hline \multicolumn{2}{|c|}{$\begin{array}{c}\text { Extinción } \\
\text { portátil }\end{array}$} & \multicolumn{5}{c|}{$\begin{array}{c}\text { Extinción } \\
\text { fija }\end{array}$} \\
\hline \multirow{2}{*}{ Peso } & \multicolumn{2}{|c|}{0,14} & \multicolumn{5}{c|}{0,26} \\
\cline { 2 - 8 } & NE & OT & AR & CR & OT & CG & RE \\
\hline Peso & 0,67 & 0,33 & 0,24 & 0,16 & 0,16 & 0,19 & 0,25 \\
\hline
\end{tabular}

La escala de puntuación propuesta para cada uno de los criterios valorados en cada tecnología para obtener la clasificación de los clientes fue 1, 4, 7 y 10 , ya que al analizar todos los criterios, los mismos estaban controlados a 4 niveles.

\section{Paso 6. Evaluación final de cada cliente}

Con los pesos de cada criterio y la utilización del método de ponderación simple (Scoring), [1-3, 5, 15-16], se obtiene la evaluación para cada una de las tecnologías; con éstas y los pesos asignados a cada una, se determina la evaluación final para cada cliente. En este caso se utilizó un enfoque de dos sumas ponderadas, es decir, se realiza una suma ponderada para cada tecnología y luego otra para la realización de la evaluación final de cada cliente, utilizando la ecuación (1) y (2) respectivamente.

$$
\begin{array}{cc}
E T_{j}=\sum_{i=1}^{r} w_{i} e_{i j} & \forall j \\
C C_{k}=\sum_{j=1}^{m} w_{j k} E T_{j} & \forall j
\end{array}
$$


donde:

$E T_{j}$ : evaluación de la tecnología $j$.

$w_{i}$ : ponderación o pesos del criterio $i$.

$e_{i j}$ : evaluación de la tecnología $j$ con el criterio $i$.

$r$ : cantidad de criterios.

$C C_{k}$ : clasificación integral del cliente $k$.

$w_{j k}$ : ponderación o pesos de la tecnología $j$ para el cliente $k$.

$E T_{j}$ : evaluación de la tecnología $j$.

$m$ : cantidad de tecnologías.

Paso 7. Determinación de la escala y la clasificación de los clientes

Con la evaluación obtenida en el paso anterior y la escala que aparece en la Tabla 4 se obtiene la clasificación de los clientes.

Tabla 4. Rangos para la clasificación del cliente.

\begin{tabular}{|c|c|}
\hline Rango del $\mathbf{C C}_{\mathbf{k}}$ & Clasificación del cliente \\
\hline$C C_{k} \geq 3,41$ & $\mathrm{~A}$ \\
\hline $1,41-3,4$ & $\mathrm{~B}$ \\
\hline$C C_{k} \leq 1,4$ & $\mathrm{C}$ \\
\hline
\end{tabular}

\section{Fábrica de helados}

En este caso se exponen solamente los resultados de la aplicación de los pasos 2, 6 y 7. En el paso 2 se presenta el árbol jerárquico obtenido del trabajo grupal, en la Figura 4 se muestra el mismo.

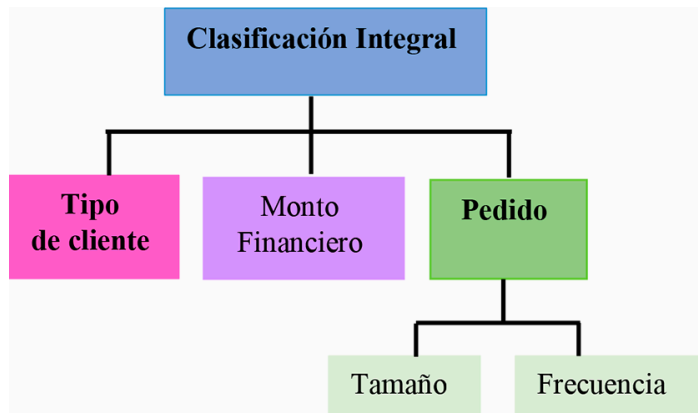

Figura 4. Árbol jerárquico de los criterios de la fábrica de helados.

\section{Paso 6. Evaluación final de cada cliente}

La agrupación de los clientes se realiza teniendo en cuenta los pesos asignados a cada criterio y la evaluación de cada uno de los clientes para cada criterio, se utiliza la expresión 1, que representa el método de ponderación simple.

Paso 7. Determinación de la escala y la clasificación de los clientes

En el caso de la fábrica de helados se propone utilizar la escala que se muestra en la Tabla 6.

\section{RESULTADOS}

En la entidad 1, se validó el procedimiento propuesto con una muestra de 60 clientes, de los cuales el 99\% posee extinción portátil, el 25\% extinción fija, el 37\% protecciones eléctricas y el 58\% seguridad electrónica.

Los resultados de la aplicación del nuevo sistema de clasificación integral, permitieron concluir que el $55 \%$ de los clientes obtuvo clasificación B, el 25\% clasificación C y el $20 \%$ clasificación A, como se muestra en la Figura 5.

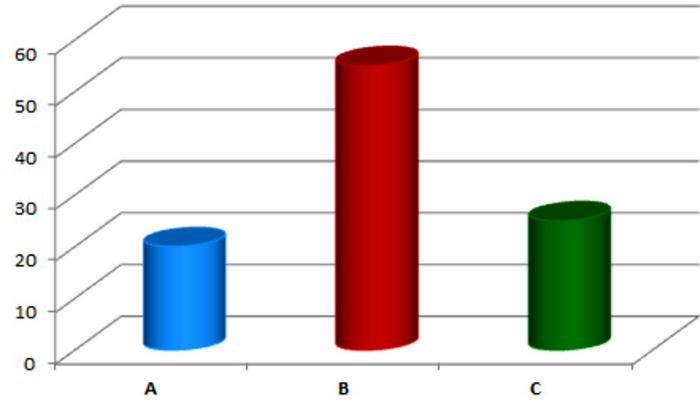

Figura 5. Composición de los clientes.

Con la clasificación integral de los clientes derivados de la aplicación del procedimiento propuesto para un día de trabajo, se obtuvo el plan de mantenimiento, los resultados del plan comparado con el anterior se muestran en la Tabla 5.

Tabla 5. Resultados con la clasificación de los clientes.

\begin{tabular}{|l|c|c|}
\cline { 2 - 3 } \multicolumn{1}{c|}{} & Antes & Después \\
\hline Km recorridos & 1219 & 713,11 \\
\hline Consumo de combustible (litros) & 101,6 & 59,43 \\
\hline Clientes visitados & 8 & 15 \\
\hline
\end{tabular}

Para la implementación del procedimiento, se diseñó una hoja de trabajo en EXCEL, que permite 
automáticamente introducir todas las evaluaciones de cada cliente para obtener de forma rápida y eficiente la clasificación integral de los mismos.

En la entidad 2 el agrupamiento obtenido de los clientes en la fábrica de helados permitió establecer un plan de distribución priorizando aquellos que se encuentran en los grupos A y B, si la capacidad de transportación y la cantidad de productos supera la demanda se pasará a satisfacer a los del grupo C, sino estos se quedarán para el siguiente día proponiéndose para éstos un nivel de servicio del $85 \%$ lo que se establecerá en el contrato correspondiente.

La Tabla 6 y la Figura 6 muestran una comparación de los resultados antes y después de la aplicación del procedimiento.

Tabla 6. Comparación de los resultados.

\begin{tabular}{|l|r|c|}
\hline Resultados mensual & \multicolumn{1}{|c|}{ Antes } & Después \\
\hline Ingreso Total (CUC) & 6117183 & 6152530,1 \\
\hline Costo Total (CUC) & 5122189 & 5116718,6 \\
\hline Utilidad & 994993,94 & 1035582,4 \\
\hline
\end{tabular}

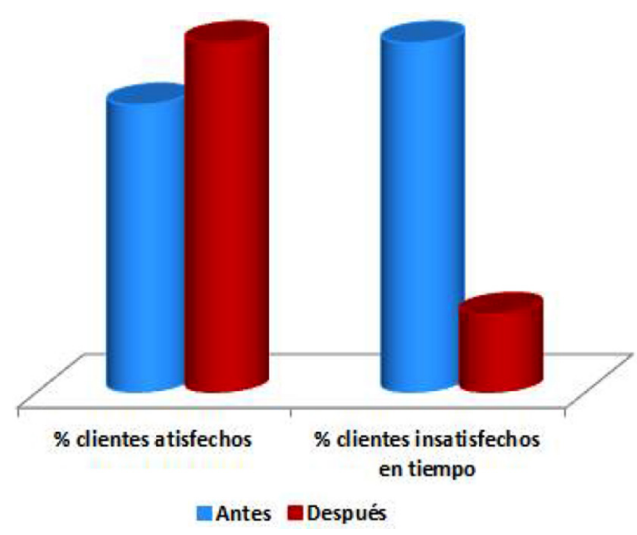

Figura 6. Comparación de algunos indicadores.

Se puede observar que el uso del procedimiento en ambas entidades permitió a las organizaciones elevar su nivel de desempeño como se muestra en los indicadores obtenidos.

La introducción de herramientas matemáticas contribuyó a mejorar el proceso de toma de decisiones, corroborando lo que se plantea en la bibliografía la cual otorga importancia a la aplicación de estas técnicas.
La clasificación actual de los clientes en las entidades objeto de estudio se realizaba de forma empírica, sin utilizar herramientas matemáticas, además no se consideraba la interrelación de los criterios. El nuevo sistema de clasificación en la entidad 1 considera las cuatro tecnologías a las que se le brinda mantenimiento, y a través de un método Delphi, concede pesos a los criterios para posteriormente utilizando el método Scoring, otorgar una puntuación integral al cliente, estableciéndose de esta manera una única clasificación de los clientes. Un procedimiento similar se realizó en la entidad 2.

\section{CONCLUSIONES}

La clasificación de grupos de individuos (clientes, proveedores, productos etc.) es un problema que puede aparecer en cualquier entidad; el uso de herramientas que permitan elevar la eficiencia de este proceso contribuye a elevar el desarrollo de las organizaciones y por ende de los países.

El procedimiento propuesto muestra una forma rápida y eficiente para obtener esta clasificación.

La aplicación de técnicas multicriteriales, específicamente el método Scoring permitió obtener la clasificación integral de los clientes, para garantizar que se puedan planificar adecuadamente los mantenimientos y los recorridos de distribución, alcanzando mejores resultados económicos.

\section{REFRENCIAS}

[1] A. Barrera. "Diseño de un procedimiento para la planificación de servicios de mantenimiento preventivo. Aplicación y validación en SEISA". Trabajo Fin de carrera. Instituto Superior Politécnico José Antonio Echeverría. CUJAE. La Habana, Cuba. 2011.

[2] A. Maher and F. Maysam. "Classifiers consensus system approach for credit scoring". Knowledge Based Systems. Vol. 104, pp. 89-105. Julio 2016. ISSN: 09507051 DOI:10.1016/j.knosys.2016.04.013.

[3] D. Bouyssou, T. Marchant, M. Pirlot, A. Tsoukias and P. Vincke. "Evaluation and decision models: a critical perspective". Kluwer Academic Publishers International series in Operations Research \& Management Sciences Massachusetts. United States of 
America. Vol. 32, pp. 262. 2000. ISBN 978-0-7923-7250-9.

[4] E. Fernández, J. Navarro and A. Duarte. "Multicriteria sorting using a valued preference closeness relation". European Journal of Operational Research. Vol. 185, pp. 673-686. Marzo 2008. ISSN, 0377-2217.

[5] E. Fernández, J. Navarro and S. Bernal. "Multicriteria sorting using a valued indifference relation under a preference disaggregation paradigm". European Journal of Operational Research. Vol. 198, pp. 602609. Octubre 2009. ISSN, 0377-2217.

[6] E. Fernández, S. Bernal, J. Navarro and R. Olmedo. "An outranking-based fuzzy logic model for collaborative group preferences". TOP. Vol. 18, pp. 444-464. Diciembre 2010. ISSN: 1863-8279.

[7] E. Martínez y R. Beausoleil. "Enfoque Multicriterial en el Establecimiento de Estructuras de Producción". Revista de Dirección, Organización y Administración de empresas. № 23, pp. 111-119. Enero 2000. ISSN: 1132-175X.

[8] J. Hongwei and Y. Zhang. "An investigation of service quality, customer satisfaction and loyalty in China's airline market". Journal of Air Transport Management. Vol. 57, pp. 80 - 88. Octubre 2016. ISSN: 0969-6997.

[9] J. Spohrer, L. Anderson, N. Pass, T. Ager and D.Gruhl. "Service Science". Journal of Grid Computing, Special Issue on Grid Economics and Business Models. Vol. 6, Issue 3, pp. 313324. Septiembre 2008. ISSN: 1572-9184.

[10] J. Spohrer, S.L.Vargo, N. Caswell and P.P. Maglio. "The Service System is the Basic abstraction of Service Science". 41st Hawaii International Conference on System Sciences. United States of America. Enero 2008.

[11] P. Aragonés. "Técnicas de ayuda a la toma de decisiones en proyectos". Informe Técnico
Universidad Politécnica de Valencia. Valencia, España. Julio 2010.

[12] P.B. Lowry and D. Wilson. "Creating agile organizations through IT: The influence of internal IT service perceptions on IT service quality and IT agility". Journal of Strategic Information Systems. Vol. 25, pp. 211-226. Octubre 2016. ISSN: 09638687.

[13] P. Kaplan and S.R. Ranjithan. "A New MCDM Approach to Solve Public Sector Planning Problems". Proceedings of the 2007 IEEE Symposium on Computational Intelligence in Multi Criteria Decision Making. Hawaii, United States of America. Abril 2007.

[14] R. Lusch, S.L. Vargo and M. Tanniru. "Service, value networks and learning". Journal of the Academy of Marketing Science. Vol. 38, pp. 19-31. Febrero 2010. ISSN electrónico 15527824.

[15] S. Berumeny y F. Llamazares. "La utilidad de los métodos de decisión multicriterio (como el AHP) en un entorno de competitividad creciente". Revista Cuaderno Administración. Vol. 20, pp. 6587. Julio de 2007. ISSN electrónico: 1900-7205.

[16] T. Hussein, Al-Badawyand and M. Abouelela. "Predictive value of different scoring systems for critically ill patients with hospital acquired pneumonia". Egyptian Journal of Chest Diseases and Tuberculosis. Vol. 65, Issue 4, pp. 733-862. Octubre 2016. ISSN: 0422-7638.

[17] T.L. Saaty. "The Analytic Hierarchy and Analytic Network Processes for the Measurement of Intangible Criteria and for Decision-making". Figueira, Greco y Erghott (eds.). Multiple Criteria Decision Analysis: State of the Art Surveys. Springer Science and Business Media. Nueva York, United State of America. pp. 345-407. 2005. ISBN 978-3540-92828-7. 